\title{
UNITARY COLLIGATIONS \\ AND PARAMETRIZATION FORMULAS
}

\section{УНІТАРНІ ОПЕРАТОРНІ ВУЗЛИ ТА ФОРМУЛИ ПАРАМЕТРИЗАЦІЇ}

This paper aims to describe, in a rather sketchy but relatively selfcontained way, some relations between the unitary colligations, which are regarded as linear systems, and the parametrization formulas for the solutions of some interpolation problems.

Наведено опис деяких співвідношень між унітарними операторними вузлами, які розглядаються як лінійні системи, та формулами параметризації для розв'язків деяких інтерполяційнх задач.

1. Preliminary Constructions. 1. Unless otherwise specified, all spaces are assumed to be separable complex Hilbert spaces and all subspaces are assumed to be closed; $\mathcal{C}(X, Y)$ denotes the set of all bounded linear operators from a space $X$ to a space $Y$, $\mathcal{C}(X)$ is the same as $\mathbb{C}(X, X)$, and " $\vee$ " means a "closed linear span"; $P_{E}^{X} \equiv P_{E}$ denotes the orthogonal projection onto the subspace $E$ of $X$ and $i_{E}^{X} \equiv i_{E}$ denotes the inclusion of $E$ in $X$.

Let $T \in \mathbb{C}\left(B_{1}, B_{2}\right)$ be a contraction (i.e., $\left.\|T\| \leq 1\right)$. There exist a space $F$ and two isometries $\rho_{1}: B_{1} \rightarrow F$ and $\rho_{2}: B_{2} \rightarrow F$, which are essentially unique and such that $T=\rho_{2}^{*} \rho_{1}$ and $F=\left(\rho_{1} B_{1}\right) \vee\left(\rho_{2} B_{2}\right)$. Moreover, if $U_{j} \in \mathbb{C}\left(B_{j}\right)$ is a unitary operator, $j=1,2$, and $U_{2} T=T U_{1}$, then there exists an essentially unique unitary operator $W \in \mathbb{C}(F)$ such that $W \rho_{j}=\rho_{j} U_{j}, j=1,2$.

In fact, let $F$ be the Hilbert space generated by the linear space $B_{1} \times B_{2}$ and a positive semidefinite form $\left\langle\left(b_{1}, b_{2}\right),\left(b_{1}^{\prime}, b_{2}^{\prime}\right)\right\rangle \equiv\left\langle b_{1}, b_{1}^{\prime}\right\rangle_{B_{1}}+\left\langle T b_{1}, b_{2}^{\prime}\right\rangle_{B_{2}}+\left\langle b_{2}, T b_{1}^{\prime}\right\rangle_{B_{2}}+$ $+\left\langle b_{2}, b_{2}^{\prime}\right\rangle_{B_{2}}$, and let $\rho_{1}: B_{1} \rightarrow F$ and $\rho_{2}: B_{2} \rightarrow F$ be the isometries generated by $b_{1} \rightarrow\left(b_{1}, 0\right)$ and $b_{2} \rightarrow\left(0, b_{2}\right)$, respectively. This implies the first assertion and, for $U_{1}, U_{2}$ as above, $W$ is defined in $F$ by setting $W \rho_{j} b=\rho_{j} U_{j} b \forall b \in B_{j}, j=1,2$.

2. We say that $V$ is an isometry acting in the space $H$ if there exist subspaces $D$ and $R$ of $H$ such that $V$ is an isometry from $D$ onto $R$. In several interpolation problems, $H$ and $V$ are naturally defined so that there exists a bijection between the set of all the solutions of the problem and the set $\mathfrak{U}_{V}$ of all (essentially different) minimal unitary extensions of $V$. We say that $(U, G)$ defines an element in $\mathfrak{U}_{V}$ (and we simply write $\left.(U, G) \in \mathfrak{U}_{V}\right)$ if $U \in \mathbb{C}(G)$ is a unitary operator, $H \subset G, V=\left.U\right|_{D}$, and the minimality condition $G=\vee\left\{U^{n} H: n \in \mathbb{Z}\right\}$ holds. We set $(U, G) \approx\left(U^{\prime}, G^{\prime}\right)$ in $\mathfrak{U}_{V}$ iff there exists a unitary operator $\tau \in \mathbb{C}\left(G, G^{\prime}\right)$ such that $\tau U=U^{\prime} \tau$ and $\left.\tau\right|_{H}=I_{H}$, where $I_{H}$ is the identity in $H$.

The defect subspaces of $V$ are the orthogonal complements $N=H \theta D$ and $M=$ $=H \theta R$ of $D$ and $R$ in $H$. If $(U, G) \in \mathbb{U}_{V}$, then a unitary operator $A \in \mathbb{C}(N \oplus X$, $M \oplus X)$ is defined by setting $X=G \theta H$ and $A=\left.U\right|_{G \theta D}$. The minimality condition guarantees that $T:=\left.P_{X} A\right|_{X}$ is a completely nonunitary (c.n. u.) contraction, i.e., that there is no nontrivial subspace $Y$ of $X$ such that $\left.T\right|_{Y}$ is a unitary operator in $Y$. This 
shows how to obtain each element in $\mathfrak{U}_{V}$ : Let $X^{\prime}$ be such that $N \oplus X^{\prime}$ and $N \oplus X^{\prime}$ have the same dimension as the Hilbert spaces and let $A^{\prime} \in \mathbb{C}\left(N \oplus X^{\prime}, M \oplus X^{\prime}\right)$ be a unitary operator. We set $G^{\prime}=H \oplus X^{\prime}, U^{\prime}=V \oplus A^{\prime}, G=\vee\left\{U^{\prime n} H: n \in \mathbb{Z}\right\}$, and $U=\left.U^{\prime}\right|_{G}$. With obvious notation, $(U, G) \approx\left(U^{\prime}, G^{\prime}\right)$ in $\mathbb{U}_{V}$ iff there exists a unitary operator $\lambda \in \mathbb{C}\left(X, X^{\prime}\right)$ such that $A^{\prime}\left(I_{N} \oplus \lambda\right)=A^{\prime}\left(I_{M} \oplus \lambda\right) A$.

3 . We have just seen that the problem of extending an isometry leads naturally to the consideration of the sets $\delta:=\left\{E_{1}, E_{2}, X ; A\right\}$, where $A$ is a bounded operator from the space $X \oplus E_{1}$ to the space $E_{2} \oplus X$ and the associated operator matrix is denoted by $\left[A_{j k}\right]_{j, k=1,2}$. The set $\delta$ is called an operator colligation; $\delta$ is unitary if $A$ is a unitary operator; it is simple if, in addition, the contraction $A_{21} \equiv P_{X} A \mid X$ is c.n. $\mathrm{u}$. The colligation $\delta^{\prime}=\left\{E_{1}, E_{2}, X^{\prime} ; A^{\prime}\right\}$ is equivalent to $\delta$ iff there exists a unitary operator $\lambda \in \mathbb{C}\left(X, X^{\prime}\right)$ such that $A^{\prime}\left(\lambda \oplus I_{E_{1}}\right)=\left(I_{E_{2}} \oplus \lambda\right) A$.

Operator colligations can be regarded as discrete linear systems: If, at time $n \in \mathbb{Z}$, the internal state is $x(n) \in X$ and the system receives an input $h_{1}(n) \in E_{1}$, then it produces an output $h_{2}(n) \in E_{2}$ and the internal state changes to $x(n+1) \in X$ so that the following dynamic equations hold:

$$
h_{2}(n)=A_{11} x(n)+A_{12} h_{1}(n), \quad x(n+1)=A_{21} x(n)+A_{22} h_{1}(n) .
$$

Thus, there exists a bijection from $\mathfrak{U}_{V}$ to the set of equivalence classes of simple unitary colligations with the input space $N$ and the output space $M$; this bijection is given by the relation $\delta(U, G)=\left\{N, M, G \theta H ;\left.U\right|_{G \theta D}\right\}$ for any $(U, G) \in \mathfrak{U}_{V}$. Each class of this sort is characterized by an analytic function; we now recall its definition.

4. We say that a function $\varphi$ belongs to the set $B\left(E_{1}, E_{2}\right)$ of contractive analytic functions if $\varphi: \mathbb{D} \rightarrow \mathbb{C}\left(E_{1}, E_{2}\right)$ is an analytic function in the open unit disk $\mathbb{D}$ on the complex plane and $\varphi(z)$ is a contraction for every $z \in \mathbb{D}$. It is well known that if $z \in \mathbb{D}$ converges nontangentially to $e^{i t}$, the strong limit $\varphi\left(e^{i t}\right)$ of $\varphi(z)$ exists a.e. Assume that $m$ is the Lebesgue normalized measure in $\mathbb{T}(\equiv \partial \mathbb{D})$ and $E$ is an arbitrary space. Let $L^{2}(E)$ be the space of measurable functions $f: \mathbb{T} \rightarrow E$ such that $\int_{\mathbb{T}}\|f\|_{E}^{2} d m<\infty$ and let $l^{2}(E)$ be the space of sequences $h: \mathbb{Z} \rightarrow E$ such that $\sum\|h(n)\|^{2}<\infty$; each $f \in L^{2}(E)$ is such that $f=\sum e_{n} \hat{f}(n)$ for $\hat{f} \in l^{2}(E)$ and $e_{n}(t) \equiv e^{i n t}$. We set $H^{2}(E)=\left\{f \in L^{2}(E): \hat{f}(n)=0\right.$ if $\left.n<0\right\}$. If $w: \mathbb{T} \rightarrow \mathbb{C}\left(E_{1}, E_{2}\right)$ is a weakly measurable function such that $\|w\|_{\infty}:=\operatorname{ess} \sup \left\|w\left(e^{i t}\right)\right\|<\infty$, we say that $w \in L^{\infty}\left(\mathbb{T} ; E_{1}, E_{2}\right)$ and the multiplication $M_{W}$ by $w$ is a bounded operator from $L^{2}\left(E_{1}\right)$ to $L^{2}\left(E_{2}\right)$ such that $\|w\|_{\infty}=\left\|M_{W}\right\|$ and $M_{W} v=\sum\left\{e_{n} \hat{w}(n) v: n \in \mathbb{Z}\right\}$ for

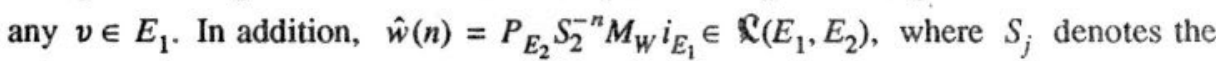
shift, i.e., $\left(S_{j} f\right)\left(e^{i t}\right) \equiv e^{i t} f\left(e^{i t}\right)$ for every $f \in L^{2}\left(E_{j}\right)$. Consider a function $w$ in $\mathbb{D}$ given by $w\left(r e^{i t}\right)=\sum\left\{r^{|n|} e^{i n t} \hat{w}(n): n \in \mathbb{Z}\right\}$ for any $r \in[0,1)$. Then $\|w\|=$ $=\sup \{\|w(z)\|: z \in \mathbb{D}\}$. If $\varphi \in \mathbb{B}\left(E_{1}, E_{2}\right)$, then $\dot{M}_{\varphi} H^{2}\left(E_{1}\right) \subset H^{2}\left(E_{2}\right)$.

Let $\delta=\left\{E_{1}, E_{2}, X ; A\right\}$ be a unitary colligation. If $x(0)=0$ and the sequence of inputs is given by $\hat{f}_{1}$ with $f_{1} \in H^{2}\left(E_{1}\right)$, the sequence of outputs is given by $\hat{f}_{2}$ with 
$f_{2}=M_{\varphi} f_{1}$, where $\varphi(z):=A_{12}+z A_{11}\left(1-z A_{21}\right)^{-1} A_{22}$ for any $z \in \mathbf{D}$. Since $A$ is unitary, we have

$$
\sum\left\{\left\|h_{2}(n)\right\|^{2}: 0 \leq n \leq m\right\}+\|x(m+1)\|^{2}=\sum\left\{\left\|h_{1}(n)\right\|^{2}: 0 \leq n \leq m\right\},
$$

and, hence, $\left\|M_{\varphi}\right\| \leq 1$. Thus, $\varphi \in \mathrm{B}\left(E_{1}, E_{2}\right)$; it is called the characteristic function of the colligation or the response function of the system; we denote this fact by setting $\varphi=$ c.f. $(\delta)$.

It is easy to see that $\delta \approx \delta^{\prime}$ implies c.f. $(\delta)=$ c.f. $\left(\delta^{\prime}\right)$. It is well known that the converse statement holds if $\delta$ and $\delta^{\prime}$ are simple unitary colligations and, moreover, that any $\varphi \in \mathrm{B}\left(E_{1}, E_{2}\right)$ is the characteristic function of such a colligation. Proofs of these two facts will be sketched in the next section. Thus, a bijection between $\mathfrak{u}_{V}$ and $\mathrm{B}(N, M)$ is established and the parametrization formulas for the solutions of interpolation problems can be obtained. This is the subject of our paper.

\section{Realization of Contractive Analytic Functions.}

1. Proposition 1. A function $\varphi$ belongs to $\mathrm{B}\left(E_{1}, E_{2}\right)$ iff there exist a unitary operator $W \in \mathbb{C}(F)$ and two isometries $\pi_{1}: E_{1} \rightarrow F$ and $\pi_{2}: E_{2} \rightarrow F$ such that $\left(\pi_{1} E_{1}\right)$ and $\left(\pi_{2} E_{2}\right)$ are wandering subspaces for $W, F=\vee\left\{\oplus\left[W^{n} \pi_{j} E_{j}: n \in \mathbb{Z}\right]\right.$ : $j=1,2\}, W^{n} \pi_{1} E_{1} \perp W^{m} \pi_{2} E_{2}$ if $n<m$, and

$$
\varphi(z)=\pi_{2}^{*}(1-z W)^{-1} \pi_{1} \quad \forall z \in \mathbf{D} .
$$

For any $\varphi \in \mathrm{B}\left(E_{1}, E_{2}\right)$, these $W, \pi_{1}$, and $\pi_{2}$ are unique up to unitary isomorphisms.

Proof. If $\varphi \in \mathbb{B}\left(E_{1}, E_{2}\right)$, the contraction $M_{\varphi}$ is such that $S_{2} M_{\varphi}=M_{\varphi} S_{1}$ for any $f \in L^{2}\left(E_{j}\right)$. Then it follows from subsection 1.1 that there exist a unitary operator $W \in \mathcal{C}(F)$ and isometries $\rho_{j}: L^{2}\left(E_{j}\right) \rightarrow F$ such that $M_{\varphi}=\rho_{2}^{*} \rho_{1}, W^{*} \rho_{j}=\rho_{j} S_{j}$, and $F=\vee\left\{\rho_{j} L^{2}\left(E_{j}\right): j=1,2\right\}=\vee\left\{\oplus\left[W^{n} \pi_{j} E_{j}: n \in \mathbb{Z}\right]: j=1,2\right\}$ for $\pi_{j}=\rho_{j} \mid E_{j}$. Then $\pi_{2}^{*} W^{n} \pi_{1}=0$ if $n<0$ and $\varphi(z) \equiv \sum\left\{z^{n} \pi_{2}^{*} W^{n} \pi_{1}: n \geq 0\right\}$. Thus, relation (1) holds. If $W^{\prime}, \pi_{1}^{\prime}$, and $\pi_{2}^{\prime}$ are as $W, \pi_{1}$ and $\pi_{2}$, then $\lambda\left(W^{n} \pi_{j} v\right) \equiv W^{\prime n} \pi_{j}^{\prime} v$ defines a unitary operator $\lambda \in \mathbb{C}\left(F, F^{\prime}\right)$ such that $\lambda W=W^{\prime} \lambda$ and $\lambda \pi_{j}=\pi_{j}^{\prime}, j=1,2$.

Conversely, let $\varphi$ be given by (1) with $W, \pi_{1}$, and $\pi_{2}$ as in the statement above; for any trigonometric polynomial $f_{j}$ in $H^{2}\left(E_{j}\right)$, we have

$$
\begin{gathered}
2\left|\left\langle M_{\varphi} f_{1}, f_{2}\right\rangle\right| \leq\left\|\sum\left\{W^{-k} \pi_{1} \hat{f}_{1}(k): k \geq 0\right\}\right\|^{2}+ \\
+\left\|\sum\left\{W^{-k} \pi_{2} \hat{f}_{2}(k): k \geq 0\right\}\right\|^{2}=\left\|f_{1}\right\|^{2}+\left\|f_{2}\right\|^{2} .
\end{gathered}
$$

Thus, $\left\|M_{\varphi}\right\| \leq 1$. This implies the required result.

Now we can prove that every contractive analytic function $\varphi$ has a realization as the characteristic function of a simple unitary colligation $\delta$, i.e., $\varphi=$ c.f. $(\delta)$. This fact was proved in [9]; it is basically contained in the book by Nagy and Foias [13]. We derive it from Proposition 1, simplifying, thus, the proof given in [1].

2. Proposition 2. Let $\varphi, W, \pi_{1}$, and $\pi_{2}$ be as in Proposition 1. We set

$$
E=\left\{\oplus\left[W^{-n} \pi_{1} E_{1}: n \geq 0\right]\right\} \oplus\left\{\left\{\oplus\left[W^{m} \pi_{2} E_{2}: m \geq 1\right]\right\}, \quad X=F \theta E .\right.
$$

Let $A=\left[A_{j k}\right]_{j, k=1,2} \in \mathbb{C}\left(X \oplus E_{1}, E_{2} \oplus X\right)$ be given by $A_{11}=\left.\pi_{2}^{*}\right|_{X}, A_{12}=\pi_{2}^{*} \pi_{1}$, 
$A_{21}=\left.P_{X} W\right|_{X}$, and $A_{22}=P_{X} W \pi_{1}$. Then $\delta:=\left\{E_{1}, E_{2}, X ; A\right\}$ is a simple unitary colligation, $\varphi=$ c.f. $(\delta), W$ is a unitary dilation of $A_{21}$, which is minimal iff $\varphi$ is a pure contractive function.

Proof. Since $W\left[X \oplus\left(\pi_{1} E_{1}\right)\right]=X \oplus W\left(\pi_{2} E_{2}\right)$, the operator $A=\left[\left(\left.\pi_{2}^{*} W^{*}\right|_{W_{\pi_{2}} E_{2}}\right) \oplus\right.$ $\left.\oplus I_{X}\right] W\left[I_{X} \oplus \pi_{1}\right]$ is unitary. It follows from $\vee\left\{W^{n} E: n \in \mathbb{Z}\right\}=F$ that $A_{21}$ is c.n.u. and $\delta$ is a simple unitary colligation. Since $P_{E} W X \subset W \pi_{2} E_{2}$, we have $W^{n} P_{E} W X \perp X$ for any $n \geq 0$ and $\left.P_{X} W^{n+1}\right|_{X}=\left(\left.P_{X} W\right|_{X}\right)^{n+1}$, i.e., $W$ is a unitary dilation of $A_{21}$. Since

$$
F \theta \vee\left\{W^{n} E, n \in \mathbb{Z}\right\}=\oplus\left\{W^{n}\left[\left(\pi_{1} E_{1}\right) \cap\left(\pi_{2} E_{2}\right)\right]: n \in \mathbb{Z}\right\}
$$

and $\varphi(0)=\pi_{2}^{*} \pi_{1}$, we conclude that $W$ is a minimal dilation iff $\|\varphi(0) h\|<\|h\|$ for every nonzero $h \in E_{1}$, i.e., iff $\varphi$ is a pure contractive function. We have $W^{n} P_{E} W X \perp X$ for any $n \geq 0, \pi_{2}^{*}=\pi_{2}^{*} P_{\pi_{2}} E_{2}$, and $P_{E} W^{n+1} \pi_{1} E_{1} \oplus\left[W^{m} \pi_{2} E_{2}: m \geq 1\right]$ if $n \geq 0$; consequently,

$$
A_{12}+z A_{11}\left(I-z A_{21}\right)^{-1} A_{22}=\pi_{2}^{*} \pi_{1}+\pi_{2}^{*} \sum\left\{z^{n} P_{X} W^{n}: n>0\right\} \pi_{1}=\varphi(z) .
$$

3. The approximately controlled subspace of a colligation $\delta=\left\{E_{1}, E_{2}, X ; A\right\}$ is $X_{c}:=\vee\left\{A_{21}^{n} A_{22} E_{1}: n \geq 0\right\}$, its approximately observable subspace is defined as follows: $X_{o b}:=\vee\left\{A_{21}^{* n} A_{11}^{*} E_{2}: n \geq 0\right\}$. By induction, one can find that $X \theta\left[X_{c} \vee X_{o b}\right]=$ $=L:=\left\{x \in X: A^{n} x=A_{21}^{n} x, A^{* n} x=A_{21}^{* n} x \forall n>0\right\}$. Moreover, $A L \subset L$ and, by duality, $A L=L$. This implies that $\delta$ is simple iff $X=X_{c} \vee X_{o b}$. This fact and the interpretation of the colligations as systems give a direct proof of the assertion that simple unitary colligations with the same characteristic function are equivalent.

Thus, we see that $\mathfrak{u}_{V} \leftrightarrow \mathrm{B}(N, M)$; this is the fact that we learned by reading [7, 8]. More precisely, it is stated in the following theorem:

4. Theorem 1. Let $V$ be an isometry acting in $H$ with domain $D$ and defect subspaces $N$ and $M$. A bijection between the set $\mathbb{U}_{V}$ of equivalence classes of minimal unitary extensions of $V$ and the set $\mathrm{B}(N, M)$ of contractive analytic functions can be defined as follows:

(i) Given $(U, G) \in \mathbb{U}_{V}$, we set $X=G \theta H$ and let $\varphi \equiv \varphi(U, G) \in \mathbb{B}(N, M)$ be the characteristic function of the colligation $\left\{N, M, X ;\left.U\right|_{X \oplus N}\right\}$, i.e.,

$$
\varphi(z)=\left.P_{M} U\right|_{N}+\left.\left.z P_{M} U\right|_{X}\left(I-\left.z P_{X} U\right|_{X}\right)^{-1} z P_{X} U\right|_{N}
$$

(ii) given $\varphi \in \mathbb{B}(N, M)$, let the unitary operator $W \in \mathbb{X}(F)$ and the isometries $v: L^{2}(N) \rightarrow F$ and $\mu: L^{2}(M) \rightarrow F$ be such that $M_{\varphi}=\mu^{*} v, W^{*} v=v S$, $W^{*} \mu=\mu S$ (S is a shift in the corresponding space), and $F=\vee\left[L^{2}(N)\right] \vee \mu\left[L^{2}(M)\right]$. We set

$$
E=\left\{\oplus\left[W^{-n}(v N): n \geq 0\right]\right\} \oplus\left\{\left\{\oplus\left[W^{m}(\mu M): m \geq 1\right]\right\}, \quad X=F \theta E,\right.
$$

and let $A \in \mathbb{C}\left(X \oplus E_{1}, E_{2} \oplus X\right)$ be given by $A(v, x)=\left[\mu^{*}(v v+x), P_{X} W(v v+x)\right]$ for any $v \in N$ and $x \in X$. If $G=H \oplus X$ and $U=V \oplus A$, then $(U, G) \in \mathbb{U}_{V}$ and $\varphi(U, G)=\varphi$. 
3. Description of All the Solutions of a General Interpolation Problem. 1. Let subspaces $B_{1} \subset L^{2}\left(E_{1}\right)$ and $B_{2} \subset L^{2}\left(E_{2}\right)$ be such that $E_{1} \subset B_{1} \subset S_{1}^{-1} B_{1}$ and $S_{2}^{-1} E_{2} \subset B_{2} \subset S_{2} B_{2}$. If $A \in \mathbb{C}\left(B_{1}, B_{2}\right)$ is such that $\left.A S_{1}\right|_{B_{1}}=P_{B_{2}} S_{2} A$, then we set $\mathcal{F}_{A}=\left\{w \in L^{\infty}\left(\mathbb{T} ; E_{1}, E_{2}\right):\left.P_{B_{2}} M_{W}\right|_{B_{1}}=A,\|w\|_{\infty}=\|A\|\right\}$.

For $B_{1}=H^{2}\left(E_{1}\right)$ and $B_{2}=H_{-}^{2}\left(E_{2}\right):=L^{2}\left(E_{2}\right) \theta H^{2}\left(E_{2}\right)$, the assertion $\mathfrak{F}_{A} \neq \varnothing$ is the Nehari-Page theorem (see [14]). If $K \subset H^{2}(E)$ is such that $S\left[H^{2}(E) \theta K\right] \subset$ $\subset H^{2}(E) \theta K$, the same assertion with $B_{1}=H^{2}(E)$ and $B_{2}=H_{-}^{2}(E) \oplus K, E_{1}=E_{2}=E$, gives the Sarason general interpolation theorem [15].

In general, the assertion $\widetilde{J}_{A} \neq \varnothing$ originates from the Nagy - Foias commutant lifting theorem (see [13] or [11]). The latter can be proved by the method in [2] giving a direct description of $\mathfrak{F}_{A}$ [3], which is sketched below for completeness.

Without loss of generality, assume that $\|A\|=1$. Let the space $H$ and the isometries $\lambda_{j} B_{j} \rightarrow H, j=1,2$, be such that $A=\lambda_{2}^{*} \lambda_{1}$ and $H=\left(\lambda_{1} B_{1}\right) \vee\left(\lambda_{2} B_{2}\right)$. Then $V\left(\lambda_{1} S_{1} b_{1}+\lambda_{2} b_{2}\right)=\lambda_{1} b_{1}+\lambda_{2} S_{2}^{-1} b_{2}$, for any $b_{1} \in B_{1}$ and $b_{2} \in B_{2}$, defines an isometry $V$ acting in $H$. If $(U, G) \in \mathbb{U}_{V}$, an isometric extension $\rho_{j}: L^{2}\left(E_{j}\right) \rightarrow G$ of $\lambda_{j}$ is defined by $\rho_{j} S_{j}^{n} b=U^{-n} \lambda_{j} b$, for any $n \in \mathbb{Z}$ and $b \in B_{j}$, and $\rho_{j} S_{j}=U^{*} \rho_{j}, j=1,2$. Since $S_{2} \rho_{2}^{*} \rho_{1}=\rho_{2}^{*} \rho_{1} S_{1}$, there exists $w \equiv w(U, G) \in L^{\infty}\left(\mathbb{T} ; E_{1}, E_{2}\right)$ such that $M_{w}=$ $\rho_{2}^{*} \rho_{1}$; in this case, $P_{B_{2}} M_{w} i_{B_{1}}=\left(\rho_{2} i_{B_{2}}\right)^{*}\left(\rho_{1} i_{B_{1}}\right)=A$ and $1 \geq\|w\|_{\infty} \geq \geq\|A\|=1$. Thus, $\mathfrak{\mathcal { F }}_{A} \neq \varnothing$.

2. A bijection from $\mathfrak{U}_{V}$ onto $\mathfrak{F}_{A}$ is defined by $(U, G) \rightarrow w(U, G)$. In fact, if $(\dot{U}$, $G) \in \mathbb{U}_{V}$, then $G=\rho_{1}\left[L^{2}\left(E_{1}\right)\right] \vee \rho_{2}\left[L^{2}\left(E_{2}\right)\right]$. Hence, in obvious notation, we have $(U, G) \approx\left(U^{\prime}, G^{\prime}\right)$ in $\mathfrak{U}_{V}$ iff $\rho_{2}^{*} \rho_{1}=\rho_{2}^{\prime *} \rho_{1}^{\prime}$. If $w \in \mathcal{F}_{A}$, there exist a unitary operator $U \in \mathbb{C}(G)$ and isometries $\left.\rho_{j}: L^{2}\left(E_{1}\right)\right] \rightarrow G, j=1,2$, such that $M_{w}=\rho_{2}^{*} \rho_{1}$, $G=\rho_{1}\left[L^{2}\left(E_{1}\right)\right] \vee \rho_{2}\left[L^{2}\left(E_{2}\right)\right], \rho_{j} S_{j}=U^{*} \rho_{j}$, and $\left(\rho_{2} i_{B_{2}}\right)^{*}\left(\rho_{1} i_{B_{1}}\right)=A$. Thus, we may assume that $\rho_{j} i_{B_{j}}=\lambda_{j}$, and then it is clear that $(U, G) \in \mathbb{U}_{V}$. The assertion follows.

For $w=w(U, G)$, we have $\hat{w}(n)=P_{E_{2}} S_{2}^{-n} \rho_{2}^{*} \rho_{1} i_{E_{1}}=P_{E_{2}} S_{2} \lambda_{2}^{*} P_{H} U^{n+1} i_{H} \lambda_{1} i_{E_{1}}$. If $n<0$, then $\hat{w}(n)=P_{E_{2}} S_{2} P_{B_{2}} M_{w} i_{B_{1}} S_{1}^{-n-1} i_{E_{1}}=P_{E_{2}} S_{2} A S_{1}^{-n-1} i_{E_{1}}$; therefore, $w_{0}\left(r e^{i t}\right):=\sum\left\{r^{|n|} e^{i n t} \hat{w}(n): n<0\right\}=\bar{z} P_{E_{2}} S_{2} A\left(I-\bar{z} S_{1}\right)^{-1} i_{E_{1}}, z=r e^{i t}$. Let $\pi_{1}:$ $E_{1} \rightarrow H$ and $\pi_{2}: E_{2} \rightarrow H$ be the isometries given by $\pi_{1}=\lambda_{1} i_{E_{1}}$ and $\pi_{2}=\lambda_{2} S_{2}^{*} i_{E_{2}}$. Thus,

$\mathcal{F}_{A}=\left\{w \in L^{\infty}\left(\mathbb{T} ; E_{1}, E_{2}\right): w(z)=w_{0}(z)+\pi_{2}^{*} P_{H} U(I-z U)^{-1} i_{H} \pi_{1},(U, G) \in \mathbb{U}_{V}\right\}$.

3. For any unitary operator $U \in \mathbb{C}(G)$ and a subspace $L$ of $G$, we set $f_{U, L}(z)=$ $=P_{L}(I-z U)^{-1} i_{L}, z \in \mathbb{D}$. Let $S^{(U, L)}$ be the characteristic function of the unitary colligation $\delta^{(U, L)}:=\{L, L, G \theta L ; U\}$, i.e.,

$$
\left.S^{(U, L)}(z) \equiv P_{L} U\right|_{L}+\left.\left.z P_{L} U\right|_{L^{\perp}}\left(I_{L^{\perp}}-\left.z P_{L^{\perp}} U\right|_{L^{\perp}}\right)^{-1} P_{L^{\perp}} U\right|_{L}
$$

Then

$$
f_{U, L}(z)=\left[I_{L}-z S^{(U, L)}(z)\right]^{-1} \quad \forall z \in \mathbb{D},
$$

which follows from $\left.\sum\left\{\left(P_{L^{\perp}} U\right)^{m-j} P_{L} U^{j}\right): 0 \leq j \leq m\right\}=U^{m} \quad \forall m \geq 0$. 
If $V$ is an isometry acting in $H$, a generalized resolvent of $V$ is $f_{U, H}$ for $(U, G) \in \mathfrak{U}_{V}$. If $\varphi \in \mathfrak{B}(N, M)$ and $(U, G) \in \mathfrak{U}_{V}$ are related as in subsection 1.4, then the Chumakin formula (see [10]) implies that

$$
f_{U, H}(z)=\left\{I_{H}-z\left[V P_{D}+\varphi(z) P_{N}\right]\right\}^{-1} \quad \forall z \in \mathbf{D},
$$

Since $V P_{D}+\varphi(z) P_{N}$ is the characteristic function of the colligation $\delta^{(U, H)} \equiv\{D \oplus N$, $R \oplus M, G \theta H ; U\}$, relation (3) follows from (2).

Thus, if $N$ and $M$ are the defect subspaces of the isometry $V$ defined in (2), a parametrization of $\left\{w \in \mathfrak{F}_{A}\right\}$ by means of $\{\varphi \in \mathbb{B}(N, M)\}$ is given by

$$
w(z)=\bar{z} P_{E_{2}} S_{2} A\left(1-\bar{z} S_{1}\right)^{-1} i_{E_{1}}+\pi_{2}^{*}\left[V P_{D}+\varphi(z) P_{N}\right]\left\{I_{H}-z\left[V P_{D}+\varphi(z) P_{N}\right]\right\}^{-1} \pi_{1} \text {. }
$$

4. On the Arov - Grossman Formula. 1 . Let $V$ be an isometry as in subsection 1.2. A unitary extension $B \in \mathbb{C}(H \oplus M, N \oplus H)$ of $V$ is given by $B(h, m)=$ $=\left(P_{N} h, m+V P_{D} h\right)$ for any $z \in H, m \in M$. If $L$ is a subspace of $H$ and $L^{\perp}=$ $=H \theta L$, we set $\delta^{(V, L)}=\left\{L \oplus M, N \oplus L, L^{\perp}, B\right\}$ and $S^{(V, L)}=$ c.f. $\left[\delta^{(V, L)}\right]$. Then $S^{(V, L)}=\left[S_{j k}\right]_{j k=1,2} \in \mathrm{B}(L \oplus M, N \oplus L)$ with

$$
\begin{gathered}
S_{11}(z)=\left.P_{N}\left(I-z P_{L^{\perp} V P_{D}}\right)^{-1}\right|_{L^{\prime}}, \quad S_{12}(z)=z P_{N}\left(I-z P_{L^{\perp} V P_{D}}\right)^{-1} P_{\left.L^{\perp}\right|_{M}}, \\
S_{12}(z)=P_{L} V P_{D}\left(I-z P_{L} \perp V P_{D}\right)^{-1}, \quad S_{22}(z)=\left.P_{L}\left(I-z V P_{D} P_{L} \perp\right)^{-1}\right|_{M} \quad \forall z \in \mathbb{D} .
\end{gathered}
$$

A theorem stated in [7] says that if $(U, G) \in \mathfrak{U}_{V}$ and $\varphi \in \mathbb{B}(N, M)$ are related as in Theorem 1, the characteristic function $S^{(U, L)}$ of $\delta^{(U, L)}:=\{L, L, G \theta L, U\}$ is given by

$$
S^{(U, L)}(z)=S_{12}(z)+S_{22}(z) \varphi(z)\left[I-S_{12}(z) \varphi(z)\right]^{-1} S_{11}(z) \quad \forall z \in \mathbb{D} .
$$

A proof of (4) is given in [8]. Another one can be found in [12], where it is connected with the description of all the liftings à la Nagy - Foias. An alternative proof was sketched in [4] and originates from the following statement.

2. Proposition 3. Let $\alpha=\left\{Y_{1} \oplus K, K \oplus Y_{2}, Y ; \tau\right\}$ be a unitary colligation such that $\left.P_{K} \tau\right|_{K}=0$. We set $\Sigma=\left[\Sigma_{k j}\right]_{j, k=1,2}=$ c.f. $(\alpha)$ and define $\omega: Y \oplus Y_{1} \rightarrow$ $\rightarrow Y_{2} \oplus Y$ by $\omega\left(y, y_{1}\right)=P_{Y_{2} \oplus Y} \tau\left[y, y_{1}, P_{K} \tau\left(y, y_{1}, 0\right)\right]$ for any $\left(y, y_{1}\right) \in Y \oplus Y_{1}$. Then (i) $\left[I-\Sigma_{12}(z)\right]$ is invertible in $\mathbb{C}(K)$ for all $z \in \mathbb{D}$; (ii) $\omega$ is unitary; (iii) the characteristic function $\sigma$ of $\left\{Y_{1}, Y_{2}, Y\right.$; $\left.\omega\right\}$ is given by

$$
\sigma(z)=\Sigma_{21}(z)+\Sigma_{22}(z)\left[I-\Sigma_{12}(z)\right]^{-1} \Sigma_{11}(z), \forall z \in \mathbb{D} .
$$

Proof. Since $\Sigma_{21}(0)=\left.P_{K} \tau\right|_{K}=0$, we have $\left\|\Sigma_{21}(z)\right\| \leq|z|$ for all $z \in \mathbb{D}$ and statement (i) is valid.

Clearly, $Y \oplus Y_{1} \supset \tau^{-1} K$ and $Y \oplus Y_{2} \supset \tau K$; since $\omega\left(\tau^{-1} K\right)=\tau K$ for all $k \in K$ and $\omega\left(y, y_{1}\right)=\tau\left(y, y_{1}, 0\right)$ for all $\left(y, y_{1}\right) \in\left[\left(Y \oplus Y_{1}\right) \theta \tau^{-1} K\right]$, we get statement (ii).

Let $\left\{y_{2}(n): n \geq 0\right\} \subset Y_{2}$ be the outputs of $\left\{Y_{1}, Y_{2}, Y ; \omega\right\}$ for $y(0)=0$ and let the inputs $\left\{y_{1}(n): n \geq 0\right\} \subset Y_{1}$ be such that $y_{1}(n)=0$ if $n>0$. Then $f(z):=$ $:=\Sigma\left\{z^{n} y_{2}(n): n \geq 0\right\}=\sigma(z) y_{1}(0)$. We set $k(0)=P_{K} \tau\left[y(0), y_{1}(0), 0\right], y(n+1)=$ $=P_{K} \tau\left[y(n), y_{1}(n), k(n)\right]$, and $k(n+1)=P_{K} \tau\left[y(n+1), y_{1}(n+1), 0\right]$. Then $\alpha$ 
answers to the inputs $\left\{y_{1}(n), k(n)\right\}$ by the outputs $\left\{k(n), y_{2}(n)\right\}$ and, thus, $g(z):=$ $=\Sigma\left\{z^{n} k(n): n \geq 0\right\}$ is such that $\Sigma_{11}(z) y_{1}(0)+\Sigma_{12}(z) g(z)=g(z)$ and $\Sigma_{21}(z) y_{1}(0)+$ $+\Sigma_{22}(z) g(z)=f(z)$. This yields statement (iii).

3. With the notation of subsection 4.1, let $\delta^{\prime}=\left\{L \oplus N, L \oplus M, X ; A^{\prime}\right\}$ be the "direct sum" of $\left\{L, L,\{0\} ; I_{L}\right\}$ and $\delta=\{N, M, X ; A\}$, where $A$ is the restriction of $U$ to $G \theta D$; hence, $\varphi=$ c.f. $(\delta)$; i.e., $A^{\prime} \in \mathbb{C}(X \oplus L \oplus N, L \oplus M \oplus X)$ is given by $A^{\prime}(x, l, n) \equiv[l, A(x, n)]$. Let $\alpha=\left\{L \oplus N, N \oplus L, L^{\perp} \oplus X ; \tau\right\}$ be the product of $\delta^{\prime}$ and $\delta^{(V, L)}$, i.e., $\tau=\left(B \oplus I_{X}\right)\left(I_{L^{\perp}} \oplus A^{\prime}\right)$. Then $\Sigma=\left[\Sigma_{j k}\right]_{j k=1,2}:=$ c.f. $(\alpha)$ is the product of $S^{(V, L)}$ and c.f. $\left(\delta^{\prime}\right)$. Thus, $\Sigma_{11}=S_{11}, \Sigma_{12}=S_{12} \varphi, \Sigma_{21}=S_{21}$, and $\Sigma_{22}=$ $=S_{22} \varphi$. We may apply Proposition 3 with $Y_{1}=Y_{2}=L, K=N$, and $Y=L^{\perp} \oplus X$. Since $\tau(g, v)=\left(P_{N} g,\left[U\left(P_{X}+P_{D}\right) g+v\right]\right)$ for any $g \in G$ and $v \in N$, we have $\omega(g)=P_{G}\left[\tau g, P_{N} \tau(g, 0)\right]=P_{G}\left(\tau g, P_{N} g\right)=U g$ for any $g \in G$, consequently $\left\{Y_{1}, Y_{2}, Y ; \omega\right\}=\left\{L, L, L^{\perp} \oplus X ; U\right\} \equiv \delta^{(l l, L)}$. This implies (4).

4. A mapping from $\mathrm{B}(U, M)$ onto $\left\{S^{(U, L)}:(U, G) \in \mathbb{U}_{V}\right\}$ is given by (4); it is a bijection when $D \vee L=R \vee L=H$. In fact, if $(U, G),\left(U^{\prime}, G^{\prime}\right) \in \mathbb{U}_{V}$ are such that $S^{(U, L)}=S^{\left(U^{\prime}, L\right)}$, then $\left.P_{L} U^{m}\right|_{L}=\left.P_{L} U^{\prime m}\right|_{L} \forall m \geq 0$ because $\left.P_{L}(I-z U)^{-1}\right|_{L} \equiv\left[I_{L}-\right.$ - $\left.z S^{(U, L)}(z)\right]^{-1}$; now, $\left.P_{H} U^{m}\right|_{H}$ is determined by $\left.P_{H} U^{m}\right|_{D},\left.P_{R} U^{m}\right|_{L}$ and $\left.P_{L} U^{m}\right|_{L}$. Since $\left.P_{H} U^{m}\right|_{D}=\left(\left.P_{H} U^{m-1}\right|_{H}\right) V$ and $\left.P_{R} U^{m}\right|_{H}=\left.V P_{D} P_{H} U^{m-1}\right|_{H}$ if $m>0$, we conclude by induction that $\left.P_{H} U^{m}\right|_{H}=\left.P_{H} U^{\prime m}\right|_{H} \forall m \geq 0$; thus, $(U, G) \approx\left(U^{\prime}, G^{\prime}\right)$.

5. We now apply formula (4) to the description of the set $\mathfrak{F}_{A}$ defined in subsection 3.1 in the notation of Section 3. We set $\pi_{1}=\lambda_{1} i_{E_{1}}$ and $\pi_{2}=\lambda_{2} S_{2}^{*} i_{E_{2}}$, as in subsection 3.2, and $L=\left(\pi_{1} E_{1}\right) \vee\left(\pi_{2} E_{2}\right)$. Then $\pi_{2}^{*} P_{H} U(I-z U)^{-1} i_{E} \pi_{1}=\pi_{2}^{*} P_{L} U(I-$ $-z U)^{-1} i_{L} \pi_{1}=\pi_{2}^{*} S^{(U, L)}(z)\left[I_{E}-z S^{(U, L)}(z)\right]^{-1} \pi_{1}$. In subsection 3.2, we have seen that $\mathcal{F}_{A}=\left\{w \in L^{\infty}\left(\mathbb{T} ; E_{1}, E_{2}\right): w(z)=w_{0}(z)+\pi_{2}^{*} P_{H} U(I-z U)^{-1} i_{H} \pi_{1}, \quad(U, G) \in \mathbb{U}_{V}\right\}$. Thus, with $\left[S_{j k}\right]_{j, k=1,2}$, as in subsection 4.1 , we obtain from (4) the following parametrization formula :

$$
\begin{gathered}
\mathcal{F}_{A}=\left\{w \in L^{\infty}\left(\mathbb{T} ; E_{1}, E_{2}\right): w(z)=w_{0}(z)+\pi_{2}^{*} S(z)\left[l_{L}-z S(z)\right]^{-1} \pi_{1},\right. \\
\left.S=S_{21}+S_{22} \varphi\left[I_{N}-S_{12} \varphi\right]^{-1} S_{11}, \varphi \in \mathbb{B}(N, M)\right\} .
\end{gathered}
$$

By comparing it with the relation given in subsection 3.3, we note that if $E_{1}$ and $E_{2}$ have finite dimension, the same happens with $L$ (in fact, $\operatorname{dim} L \leq \operatorname{dim} E_{1}+$ $+\operatorname{dim} E_{2}$ ), while, in general, $\operatorname{dim} H=\infty$.

Appendix: On the Schur Analysis. To conclude, we indicate certain relations, to be developed elsewhere, between our subject and the Schur analysis (concerning this topic and the operator theory, see [11]).

With each $(U, G) \in \mathbb{U}_{V}$, a sequence of contractions $\left\{\gamma_{k}: k=0\right\}$ is associated by setting $N_{0}=N, M_{0}=M, H_{p}=V\left\{U^{j} H: 0 \leq j \leq p\right\}, N_{k}=H_{k} \theta H_{k-1}, M_{k}=$ $=H_{k} \theta U H_{k-1}$, and $\gamma_{k}=\left.P_{M_{k-1}} U\right|_{N_{k-1}}$ for all $k>0$. Each $\gamma_{k}$ determines the relative 
position of the subspaces $U N_{k-1}$ and $M_{k}$. In the scalar Carathéodory-Féjer problem,

$\left\{\gamma_{k}\right\}$ is the classical sequence of the Schur parameters [6].

By definition, an $(N, M)$-choice sequence is an element of the set $\mathbb{B}(N, M)$ of sequences of contractions $\left\{\Gamma_{k}: k>0\right\}$ such that $\Gamma_{1} \in \mathbb{C}(N, M)$ and $\Gamma_{k+1} \in \mathbb{C}\left(\mathcal{D}_{\Gamma_{k}}\right.$, $\left.D_{\Gamma_{k}^{*}}\right) \forall k>0$. (If $T \in \mathbb{C}(X, Y)$ and $\|T\| \leq 1, D_{T}=\left(I-T^{*} T\right)^{1 / 2}$ and $D_{T}$ denotes the closure of $D_{T} X$ ).

A bijection between $\mathfrak{U}_{V}$ and $\mathfrak{B}(N, M)$ is obtained [5] by associating with each $(U, G) \in \mathbb{U}_{V}$ the sequence $\Gamma(U, G) \in \mathbb{B}(N, M)$ given by $\Gamma_{1}=\gamma_{1}$ and $\Gamma_{k+1}=\lambda_{1} \ldots$ $\ldots \lambda_{k} \gamma_{k+1} \rho_{k}^{*} \ldots \rho_{i}^{*} \mid D_{\Gamma_{k}} \forall k>0$, where $\rho_{k} \in \mathbb{C}\left(N_{k}, D_{\gamma_{k}}\right)$ and $\lambda_{k} \in \mathbb{C}\left(M_{k}, D_{\gamma_{k}^{*}}\right)$ are the unitary operators defined by $\left.\rho_{k}\left(U-\gamma_{k}\right)\right|_{N_{k-1}}=D_{\gamma_{k}}$ and $\left.\lambda_{k}\left(I-U_{\gamma_{k}^{*}}\right)\right|_{M_{k-1}}=$ $=D_{\gamma_{k}}$.

With each $\varphi \in \mathbb{B}(N, M)$, the Schur algorithm associates $\Theta \varphi \in \mathbb{B}\left(D_{\Gamma}, D_{\Gamma^{*}}\right)$, with $\Gamma=\varphi(0)$, defined as follows. Assume that $\delta=\{N, M, X ; A\}$ is a simple unitary colligation such that $\varphi=$ c.f. $(\delta), N_{1}$ and $M_{1}$ are the closures of $(A-\Gamma) N$ and $\left(I-A \Gamma^{*}\right) M$, respectively, $X_{1}=X \theta N_{1}$, and $A_{1}=\left.A\right|_{X}$. Then $\delta_{1}:=\left\{N_{1}, M_{1}, X_{1} ; A_{1}\right\}$ is also a simple unitary colligation. We set $\delta_{1}=$ c.f. $\left(\delta_{1}\right)$ and let $\rho \in \mathbb{C}\left(N_{1}, \mathcal{D}_{\Gamma}\right)$ and $\lambda \in \mathbb{C}\left(M_{1}, D_{\left.\Gamma^{*}\right)}\right.$ be the unitary operators given by $\left.\rho(A-\Gamma)\right|_{N}=D_{\Gamma}$ and $\lambda(I-$ $\left.-A \Gamma^{*}\right)\left.\right|_{M}=D_{\Gamma^{*}}$. Then $\mathcal{G}_{\varphi}:=\lambda \varphi_{1}(\cdot) \rho^{*}$ is such that

$$
\varphi(z)=\Gamma+z D_{\Gamma^{*}}(\widetilde{\Theta} \varphi)(z)\left[I+z \Gamma^{*}(\widetilde{\Theta} \varphi)(z)\right]^{-1} D_{\Gamma} \quad \forall z \in \mathbb{D} .
$$

By setting $f_{0}=\varphi, f_{k}=\widetilde{G} f_{k-1}$, and $\Gamma_{k}=\widetilde{\circlearrowleft} f_{k-1}(0)$ for all $k>0$, an element $\Gamma(\varphi) \in \mathfrak{B}(N, M)$ is defined.

If $(U, G) \in \mathbb{U}_{V}$ and $\varphi=\varphi(U, G)$ is as in Theorem 1, then $\Gamma(U, G)=\Gamma(\varphi)$.

1. Arocena $R$. Characteristic functions of unitary colligations and of isometries with scale subspaces // Acta cient. venez. - 1987. - 38. - P. $540-543$.

2. Arocena $R$. Unitary extensions of isometries and contractive intertwining dilations // Operator Theory: Adv. and Appl. - 1989. - 41. - P. $13-23$.

3. Arocena $R$. Some remarks on lifting and interpolation problems (To appear)

4. Arocena $R$. Commutative unitary extensions of isometries // Publ. Mat del Uruguay. - 1988. - 1. P. $67-80$.

5. Arocena $R$. Schur analysis of a class of translation invariant forms // Analysis and Partial Different. Equat. (A Collection of Papers dedicated to Mischa Cotlar (ed. C. Sadosky)). - New York, Basel: M. Dekker, 1990.

6. Arocena $R$. On a geometric interpretation of Schur parameters // Rev. Unión mat. Argent. - 1990. - 34. - P. 150 - 165 .

7. Arov D. Z., Grossman L. Z. Scattering matrices in the theory of dilations of isometric operators // Sov. Math. Dokl. - 1983. - 27. - P. 518 - 522.

8. Arov D. Z., Grossman L. Z. Scattering matrices in the theory of unitary extensions of isometric operators. - Preprint.

9. Brodskii Y. M., Shvartsman Ja. S. On invariant subspaces of contractions // Sov. Math. Dokl. 1971. - 12. - P. $1659-1663$.

10. Chumakin M. E. Generalized resolvents of isometric operators // Sib. Mat J. - 1967. $-8, \mathrm{~N}^{2} 4 .-$ P. $876-892$.

11. Foias $C$., Frazho A. E. The commutant lifting approach to interpolation problems. - Basel etc.: Birkhäuser, 1990.

12. Morán M. D. On intertwining dilations // J. Math Anal. and Appl. - 1989. - 141. - P. $219-234$.

13. Nagy B.Sz., Foias C. Harmonic analysis of operators on Hilbert space. - Amsterdam: North-Holland, 1970.

14. Nikolskii N. K. Treatise on the shift operator. - New York: Springer, 1986.

15. Sarason D. Generalized interpolation in $H^{\infty} / /$ Trans. Amer. Math. Soc.- 1967. 127. - P. 179203. 